Pak. j. sci. ind. res. Ser. A: phys. sci. 2020 63A(1) 30-39

\title{
Temperature Dependence of Volumetric and Viscometric Studies on SDS in Amino Acid Solutions
}

\author{
Muhammad Sarwar Hossain*, Bishwajit Kumar Das and Abul Khayer Morol \\ Chemistry Discipline, Khulna University, Khulna-9208, Bangladesh
}

(received February, 2018; revised April 27, 2019; accepted April 29, 2019)

\begin{abstract}
The temperature dependence studies on the binary and ternary system are very few. The densities of sodium dodecyl sulphate (SDS), L-arginine, 2-amino butyric acid in water and SDS mixed with L-arginine and 2-amino butyric acid solutions have been measured precisely at five equidistant temperatures ranging from $293.15 \mathrm{~K}$ to $313.15 \mathrm{~K}$ at an interval of $5 \mathrm{~K}$. The variety of different thermodynamics properties of SDS were observed in the low concentration region. The density of all the studied system was found to increase with the increase of molar concentration of the solution and decrease with the increase of temperature. SDS acts as structure breaker for both regions in aqueous L-arginine and 2-amino butyric acid solvent system at studied temperature.
\end{abstract}

Keywords: sodium dodecyl sulphate, amino acid, viscosity coefficient, apparent molar volume

\section{Introduction}

L-Arginine (abbreviated as Arg or R) (Debye and Anacker, 1951) is a $\alpha$-amino acid that is used in the biosynthesis of proteins (IUPAC-IUBMB, 2007). In mammals, arginine is classified as a semi-essential or conditionally essential amino acid, depending on the developmental stage and health status of the individual (Tapiero et al., 2002). Preterm infants are unable to synthesize or create arginine internally, making the amino acid nutritionally essential for them (Wu et al., 2004).

2-Aminobutyric acid is an organic compound with the formula $\mathrm{CH}_{3} \mathrm{CH}_{2} \mathrm{CH}\left(\mathrm{NH}_{2}\right) \mathrm{COOH}$. 2-Aminobutyric acid (AABA) is an isomer of the amino acid. Amino butyric acid with chemical formula $\mathrm{C}_{4} \mathrm{H}_{9} \mathrm{NO}_{2}$. There is also gamma-amino butyric acid (GABA) and beta-amino butyric acid (BABA). It is a key intermediate in the biosynthesis of ophthalmic acid, which was first isolated from calf lens.

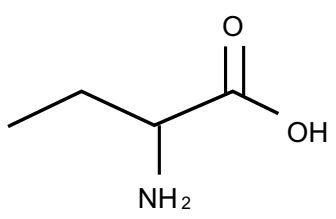

Micelles formed by surfactants dissolved in water are ubiquitous, not only as household soaps and detergents but also in drug delivery, oil recovery and environmental

*Author for correspondence; E-mail: hsarwar86@yahoo.com remediation applications (Joshua et al., 2015). The structure of micelles is very similar to the lipid bi-layer of biological membranes. The bi-layers have the same type of interactions. The lipids, however, face each other giving a double layer of lipids instead of a sphere (John and Speier, 1952). The principle is the same as the structure of micellar aggregates with a hydrophobic interior and a polar exterior. Some types of lipids can readily exchange into the cellular membrane, from the micellar aggregate. The possible micelle structure includes the spherical, lamellar and disc-like arrangement, which is shown in below (George and Harold, 1962).

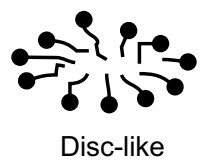

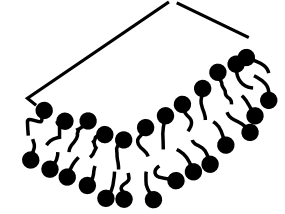

lamellar

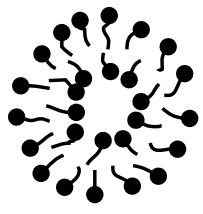

Spherical vesicle
Volumetric studies of sodium dodecyl sulfate (SDS) in aqueous and in aqueous amino acid provides great help to determine the nature of interactions and properties of this solution. It is very important because of the great applications of SDS in pharmaceuticals and biotechnological process. SDS is reported to act as a most potent protein denaturant than urea and guanidine hydrochloride (Shashank and Jagdish, 2001). SDS represents a potentially effective topical microbicide, which can also 
inhibit and possibly present infection by various enveloped and non-enveloped viruses such as the Herpes simplex viruses, HIV and the Semliki virus. In ionic surfactants, the repulsive forces originated primarily from electrostatic repulsion between the polar head groups, whereas, attractive interactions have generally been attributed to hydrophobic interactions (Niki and Jha Nand, 2011) between the nonpolar tails of the surfactant monomers. To understand the interactions of proteins with surfactants should be studied. During the past year, there have been some investigations that reported only volumetric properties of some amino acids in aqueous surfactant solutions at $\mathrm{T}=298.15 \mathrm{~K}$. However, no report is available on the physical properties of SDS in amino acid at different temperature (Xiaomei et al., 2008; Singh et al., 2004). In this paper, we report the changes in the physical properties due to the mixing of water. Solute-solvent, solute-solute, and solventsolvent have great importance in biological chemistry, physical chemistry, surface chemistry, environmental chemistry and geochemistry. The partial molar volume and viscosity B-coefficient are the important physicochemical properties to understand the interactions between molecules in solution. Thus, in continuation with our earlier work (Kabiraz et al., 2011) in the present paper we report the study of the volumetric, viscometric and the ultrasound behaviour of sodium dodecyl sulfate in water and in $0.1 \mathrm{~m} \mathrm{~L}$-arginine and 2-amino butyric acid. Using these data, also we are calculated infinite dilution apparent molar volumes, Heplar constant, viscosity B-coefficients.

\section{Materials and Methods}

In this study, sodium dodecyl sulphate (SDS) was used as a surfactant. The cmc value of SDS in water reported at around $0.0088 \mathrm{~m}$ at $25^{\circ} \mathrm{C}$. L-arginine (purity, mass fraction $\geq 0.99$ ), 2-amino butyric acid (purity, mass fraction $\geq 0.99$ ) procured from the MERCK chemical company. Supplied distilled water was used in the experiment without any redistilled and deionized. A conductivity of this deionized water was found to be $4.00 \mu \mathrm{S}$. The conductance's of electrolyte solutions were measured using the conductivity meter (model 4310 HANNA Company), which based on the resistance of the solution in a conductivity cell. At first, the conductance of water was measured a definite temperature, which was used to calibrate the conductivity meter. Then the solutions of electrolyte were used one after another to determine the conductance of the solutions at the same temperature. After the measurement of one solution, the cell and the electrode were rinsed several times with the solution of higher concentration and finally, the solution was used for the measurements. All measurements were made starting from the lowest to the highest solute concentration.

The density of the pure liquid and liquid-liquid mixtures was measured using analog process. Density determination by a Pycnometer is a very precise method (Jones and Dole, 1929). It uses a working liquid with wellknown density. An electric balance with an accuracy of $\pm 0.0001 \mathrm{~g}$ was used for weighing. The flow time of solutions was recorded by an electronic stopwatch capable of reading up to 0.01 second. A constant temperature water thermostat was used for the measurements of viscosity of the solution. The temperature of the thermostat was maintained constant to an accuracy of $\pm 0.1 \mathrm{~K}$.

The viscosities were measured at the studied temperature using an Ostwald's suspended level type viscometer which has a flow time of about $214 \mathrm{~s}$ for water at $303.15 \mathrm{~K}$. The viscometer was calibrated with water. The density and viscosity measurements were carried out in the same water bath $( \pm 0.01 \mathrm{~K})$. For the measurement of viscosity, inside of the viscometer was cleaned and washed with warm chromic acid \& distilled water respectively. So, that there was no obstruction in the capillary and the liquid could run freely without leaving any drop behind. It was then clamped vertically in the thermostatic water bath such that the upper mark of the top bulb was well below the water level. About $15 \mathrm{~min}$ were allowed for the viscometer so that the inside liquid attains the temperature of the bath. With the help of a clean rubber tube attached to the narrower limb of the viscometer, the water was sucked up above the upper mark of the bulb. The water of the bulb was then allowed to fall into capillaries and the time of fall between the two markings was noted with the help of stopwatch capable of reading up to $0.01 \mathrm{sec}$. From such three readings the meantime was taken (Masanori et al., 1993).

\section{Results and Discussion}

The measured densities of binary and ternary solutions are listed in Tables SI and Table 1, respectively as functions of concentration and temperature. The densities of all the studied systems have been found to increase with the increase in the molal concentration of binary 
Table 1. Concentration dependence of density and apparent molar volumes for SDS in $0.1 \mathrm{~m} \mathrm{~L}$-arginine and 2amino butyric acid solutions at $293.15 \mathrm{~K}, 298.15 \mathrm{~K}, 303.15 \mathrm{~K}, 308.15 \mathrm{~K}$, and $313.15 \mathrm{~K}$

\begin{tabular}{|c|c|c|c|c|c|c|c|c|c|c|}
\hline \multirow{2}{*}{$\begin{array}{l}\text { Conc./m/ } \\
\mathrm{mol} / \mathrm{kg}\end{array}$} & \multirow{2}{*}{$\begin{array}{l}\text { Density, } \rho \\
293.15 \mathrm{~K}\end{array}$} & \multirow{2}{*}{$\begin{array}{l}\mathrm{kg} / \mathrm{m}^{3} / 10^{3} \\
298.15 \mathrm{~K}\end{array}$} & \multicolumn{8}{|c|}{ Apparent molar volume, $\varphi_{\mathrm{v}} / \mathrm{m}^{3} / \mathrm{mol} / 10^{6}$} \\
\hline & & & $303.15 \mathrm{~K}$ & $308.15 \mathrm{~K}$ & $313.15 \mathrm{~K}$ & $293.15 \mathrm{~K}$ & $298.15 \mathrm{~K}$ & $303.15 \mathrm{~K}$ & $308.15 \mathrm{~K}$ & $313.15 \mathrm{~K}$ \\
\hline \multicolumn{11}{|c|}{ SDS in $0.1 \mathrm{~m}$ aqueous $\mathrm{L}$-arginine solution } \\
\hline 0.0003 & 1.003560 & 1.002357 & 1.000917 & 0.999507 & 0.998027 & 221.25 & 221.44 & 221.66 & 221.88 & 222.11 \\
\hline 0.0005 & 1.003573 & 1.002370 & 1.000930 & 0.999520 & 0.998040 & 222.89 & 223.08 & 223.31 & 223.53 & 223.77 \\
\hline 0.0020 & 1.003586 & 1.002383 & 1.000943 & 0.999533 & 0.998053 & 223.27 & 223.96 & 224.69 & 225.92 & 226.16 \\
\hline 0.0040 & 1.003682 & 1.002478 & 1.001037 & 0.999625 & 0.998145 & 223.47 & 224.40 & 224.89 & 226.12 & 226.86 \\
\hline 0.0060 & 1.003810 & 1.002604 & 1.001163 & 0.999749 & 0.998267 & 223.55 & 224.57 & 224.97 & 226.70 & 227.11 \\
\hline 0.0080 & 1.003938 & 1.002730 & 1.001289 & 0.999870 & 0.998389 & 223.82 & 224.88 & 225.24 & 226.85 & 228.73 \\
\hline 0.0100 & 1.004064 & 1.002854 & 1.001413 & 0.999992 & 0.998499 & 224.04 & 225.03 & 225.67 & 227.10 & 228.85 \\
\hline 0.0120 & 1.004189 & 1.002978 & 1.001534 & 1.000112 & 0.998617 & 224.18 & 225.12 & 225.86 & 227.42 & 228.93 \\
\hline 0.0140 & 1.004315 & 1.003103 & 1.001657 & 1.000231 & 0.998736 & 224.29 & 225.48 & 226.29 & 227.88 & 229.64 \\
\hline 0.0160 & 1.004440 & 1.003223 & 1.001775 & 1.000346 & 0.998845 & 224.39 & 225.64 & 226.50 & 227.99 & 229.94 \\
\hline 0.0180 & 1.004563 & 1.003343 & 1.001893 & 1.000463 & 0.998956 & 224.43 & 225.79 & 227.42 & 228.27 & 230.54 \\
\hline 0.0200 & 1.004688 & 1.003464 & 1.001999 & 1.000578 & 0.999062 & 224.55 & 225.95 & 227.68 & 228.82 & 230.94 \\
\hline 0.0220 & 1.004812 & 1.003585 & 1.002115 & 1.000687 & 0.999170 & 224.67 & 226.09 & 227.74 & 229.71 & 231.20 \\
\hline 0.0240 & 1.004935 & 1.003705 & 1.002234 & 1.000786 & 0.999279 & 224.95 & 226.19 & 227.97 & 230.05 & 231.44 \\
\hline \multicolumn{11}{|c|}{ SDS in $0.1 \mathrm{~m}$ aqueous 2-Amino butyric acid solution } \\
\hline 0.0003 & 1.000983 & 0.999794 & 0.998371 & 0.996735 & 0.994905 & 221.65 & 218.51 & 222.06 & 222.31 & 222.60 \\
\hline 0.0005 & 1.000990 & 0.999802 & 0.998386 & 0.996743 & 0.994920 & 235.08 & 231.36 & 219.77 & 233.88 & 220.29 \\
\hline 0.0020 & 1.001077 & 0.999885 & 0.998457 & 0.996819 & 0.994986 & 231.17 & 232.38 & 235.65 & 236.95 & 238.81 \\
\hline 0.0040 & 1.001192 & 0.999998 & 0.998572 & 0.996928 & 0.995092 & 230.87 & 232.08 & 233.34 & 235.64 & 237.50 \\
\hline 0.0060 & 1.001309 & 1.000110 & 0.998677 & 0.997033 & 0.995193 & 230.45 & 232.16 & 234.25 & 235.89 & 237.91 \\
\hline 0.0080 & 1.001421 & 1.000217 & 0.998782 & 0.997135 & 0.995292 & 230.86 & 232.82 & 234.70 & 236.38 & 238.36 \\
\hline 0.0100 & 1.001513 & 1.000308 & 0.998869 & 0.997222 & 0.995378 & 233.06 & 234.77 & 236.74 & 238.15 & 239.92 \\
\hline 0.0120 & 1.001599 & 1.000392 & 0.998953 & 0.997303 & 0.995457 & 235.09 & 236.73 & 238.42 & 239.90 & 241.60 \\
\hline 0.0140 & 1.001690 & 1.000482 & 0.999040 & 0.997386 & 0.995543 & 236.14 & 237.64 & 239.35 & 240.96 & 242.25 \\
\hline 0.0160 & 1.001777 & 1.000564 & 0.999120 & 0.997468 & 0.995617 & 237.08 & 238.74 & 240.40 & 241.72 & 243.41 \\
\hline 0.0180 & 1.001874 & 1.000659 & 0.999213 & 0.997558 & 0.995705 & 237.32 & 238.94 & 240.55 & 241.94 & 243.59 \\
\hline 0.0200 & 1.001958 & 1.000740 & 0.999292 & 0.997625 & 0.995785 & 238.20 & 239.83 & 241.41 & 243.29 & 244.16 \\
\hline 0.0220 & 1.002042 & 1.000825 & 0.999375 & 0.997705 & 0.995860 & 238.89 & 240.35 & 241.90 & 243.79 & 244.84 \\
\hline 0.0240 & 1.002122 & 1.000902 & 0.999450 & 0.997779 & 0.995932 & 239.62 & 241.10 & 242.64 & 244.43 & 245.52 \\
\hline
\end{tabular}

and ternary solutions of SDS (Fig. 1-2). The apparent molar volumes $\left(\varphi_{\mathrm{v}}\right)$ were determined from the solution densities using the following equation: (Mahendra et al., 2010)

$$
\varphi_{\mathrm{v}}=\frac{\mathrm{M}}{\rho}-1000\left(\rho-\rho_{0}\right) / \mathrm{m} \rho \rho_{0}
$$

where:

$\mathrm{M}$ is the molar mass of the solute, $\mathrm{m}$ is the molality of the solution and $\rho_{0}$ and $\rho$ are the densities of the solvent and the solution respectively. The variation of the apparent molar volume of SDS in aqueous amino acid solutions in the different concentration region shows a sudden change at a particular molality, which may be for CMC formation (Fig. 3-4). The apparent molar volume of SDS in aqueous and in aqueous amino acids may have two components, viz. the true size of the molecule and the free space between the molecules. In the pre-micellar region, the molecules exist as monomers and the monomer interaction may account for change 
of $\varphi_{\mathrm{v}}$ with concentration having the free space between the molecules. The apparent molar volume at infinite dilution, $\left(\varphi_{\mathrm{v}}^{0}\right)$ was calculated using least square fit to the linear plots of experimental values of $\varphi_{\mathrm{v}}$ versus the square root of molal concentration $(\sqrt{ } \mathrm{m})$ using the following Masson equation (Masson, 1929):

$$
\varphi_{\mathrm{v}}=\varphi_{\mathrm{v}}^{0}+\mathrm{S}_{\mathrm{v}} \sqrt{\mathrm{m}}_{\mathrm{m}}
$$

where:

$\mathrm{Sv}$ is the experimental slope, which is sometimes considered to be the volumetric pairwise interaction coefficient (Desnoyers, 2009; Hedwig et al. 1991).

Table SI. Concentration dependence of density and viscosity for different binary solutions at $293.15 \mathrm{~K}$, $298.15 \mathrm{~K}, 303.15 \mathrm{~K}, 308.15 \mathrm{~K}$, and $313.15 \mathrm{~K}$

\begin{tabular}{|c|c|c|c|c|c|c|c|c|c|c|}
\hline \multirow{2}{*}{$\begin{array}{l}\text { Conc. } / \mathrm{m} / \\
\mathrm{mol} / \mathrm{kg}\end{array}$} & \multirow{2}{*}{$\begin{array}{l}\text { Density } \\
293.15 \mathrm{~K}\end{array}$} & \multicolumn{3}{|c|}{$\rho / \mathrm{kg} / \mathrm{m}^{3} / 10^{3}$} & \multicolumn{3}{|c|}{ Viscosity, $\eta / \mathrm{kg} / \mathrm{m} / \mathrm{s} / 10^{2}$} & \multirow[b]{2}{*}{$303.15 \mathrm{~K}$} & \multirow[b]{2}{*}{$308.15 \mathrm{~K}$} & \multirow[b]{2}{*}{$313.15 \mathrm{~K}$} \\
\hline & & $298.15 \mathrm{~K}$ & $303.15 \mathrm{~K}$ & $308.15 \mathrm{~K}$ & $313.15 \mathrm{~K}$ & $293.15 \mathrm{~K}$ & $298.15 \mathrm{~K}$ & & & \\
\hline \multicolumn{11}{|c|}{ SDS in water solution } \\
\hline 0.0003 & 0.998209 & 0.997045 & 0.995644 & 0.994026 & 0.992210 & 1.014340 & 0.895246 & 0.801506 & 0.723249 & 0.656635 \\
\hline 0.0005 & 0.998221 & 0.997057 & 0.995655 & 0.994037 & 0.992220 & 1.016031 & 0.895528 & 0.801785 & 0.723559 & 0.656983 \\
\hline 0.0020 & 0.998310 & 0.997144 & 0.995740 & 0.994120 & 0.992299 & 1.019894 & 0.896284 & 0.802356 & 0.724073 & 0.657565 \\
\hline 0.0040 & 0.998425 & 0.997255 & 0.995848 & 0.994224 & 0.992402 & 1.020387 & 0.896888 & 0.802791 & 0.724470 & 0.658467 \\
\hline 0.0060 & 0.998538 & 0.997365 & 0.995955 & 0.994327 & 0.992500 & 1.024493 & 0.897626 & 0.803187 & 0.724961 & 0.659895 \\
\hline 0.0080 & 0.998647 & 0.997472 & 0.996058 & 0.994429 & 0.992601 & 1.025870 & 0.898091 & 0.803656 & 0.725319 & 0.661553 \\
\hline 0.0100 & 0.998750 & 0.997569 & 0.996155 & 0.994524 & 0.992696 & 1.026983 & 0.898973 & 0.803851 & 0.725918 & 0.662791 \\
\hline 0.0120 & 0.998851 & 0.997670 & 0.996252 & 0.994619 & 0.992789 & 1.029241 & 0.900789 & 0.805398 & 0.726403 & 0.664558 \\
\hline 0.0140 & 0.998949 & 0.997768 & 0.996348 & 0.994715 & 0.992884 & 1.034363 & 0.907256 & 0.808531 & 0.727911 & 0.666743 \\
\hline 0.0160 & 0.999048 & 0.997867 & 0.996441 & 0.994806 & 0.992975 & 1.036165 & 0.909091 & 0.811372 & 0.730039 & 0.668851 \\
\hline 0.0180 & 0.999145 & 0.997957 & 0.996536 & 0.994898 & 0.993061 & 1.039626 & 0.911422 & 0.813480 & 0.734476 & 0.671751 \\
\hline 0.0200 & 0.999237 & 0.998048 & 0.996624 & 0.994988 & 0.993153 & 1.042431 & 0.913600 & 0.816067 & 0.738761 & 0.674655 \\
\hline 0.0220 & 0.999338 & 0.998142 & 0.996718 & 0.995076 & 0.993239 & 1.046886 & 0.919078 & 0.819278 & 0.742193 & 0.677519 \\
\hline 0.0240 & 0.999426 & 0.998232 & 0.996807 & 0.995164 & 0.993323 & 1.052416 & 0.921139 & 0.820550 & 6535 & 0.679395 \\
\hline \multicolumn{11}{|c|}{ L-Arginine + water solution } \\
\hline 0.0500 & 1.000282 & 1.033917 & 1.032337 & 1.030588 & 1.028750 & 1.006771 & 0.902704 & 0.867924 & 0.781278 & 0.725081 \\
\hline 0.1000 & 1.002333 & 1.033847 & 1.032267 & 1.030525 & 1.028678 & 1.024239 & 0.933195 & 0.854117 & 0.787345 & 0.716764 \\
\hline 0.1500 & 1.004350 & 1.033796 & 1.032186 & 1.030453 & 1.028615 & 1.032139 & 0.931689 & 0.850594 & 0.780012 & 0.717917 \\
\hline 0.2000 & 1.006333 & 1.033726 & 1.032123 & 1.030385 & 1.028555 & 1.048621 & 0.942989 & 0.858069 & 0.781514 & 0.717989 \\
\hline 0.2500 & 1.008282 & 1.033666 & 1.032063 & 1.030326 & 1.028490 & 1.066327 & 0.956481 & 0.874736 & 0.798071 & 0.728988 \\
\hline 0.3000 & 1.010201 & 1.033608 & 1.031995 & 1.030268 & 1.028429 & 1.101516 & 0.972750 & 0.859617 & 0.778544 & 0.712522 \\
\hline 0.3499 & 1.012089 & 1.033544 & 1.031922 & 1.030208 & 1.028364 & 1.107268 & 0.979064 & 0.894707 & 0.810183 & 0.735871 \\
\hline 0.4000 & 1.013943 & 1.033477 & 1.031845 & 1.030145 & 1.028301 & 1.131166 & 0.986041 & 0.895382 & 0.816205 & 0.735134 \\
\hline \multicolumn{11}{|c|}{ 2-Amino butyric acid + water solution } \\
\hline 0.0500 & 0.999590 & 0.998417 & 0.997003 & 0.995378 & 0.993554 & 1.028130 & 0.908189 & 0.816891 & 0.730022 & 0.667252 \\
\hline 0.1000 & 1.000973 & 0.999789 & 0.998364 & 0.996730 & 0.994899 & 1.046381 & 0.925074 & 0.831571 & 0.744455 & 0.678392 \\
\hline 0.1500 & 1.002345 & 1.001148 & 0.999712 & 0.998070 & 0.996231 & 1.064705 & 0.941795 & 0.846727 & 0.756127 & 0.690367 \\
\hline 0.2000 & 1.003698 & 1.002487 & 1.001040 & 0.999389 & 0.997543 & 1.081946 & 0.957020 & 0.861277 & 0.769723 & 0.702224 \\
\hline 0.2500 & 1.005040 & 1.003815 & 1.002357 & 1.000696 & 0.998843 & 1.100560 & 0.973031 & 0.877715 & 0.784186 & 0.713587 \\
\hline 0.3000 & 1.006367 & 1.005128 & 1.003658 & 1.001989 & 1.000130 & 1.118012 & 0.990962 & 0.892334 & 0.797503 & 0.724949 \\
\hline 0.3499 & 1.007686 & 1.006431 & 1.004947 & 1.003269 & 1.001405 & 1.137399 & 1.006677 & 0.906820 & 0.811398 & 0.736749 \\
\hline 0.4000 & 1.008988 & 1.007715 & 1.006218 & 1.004531 & 1.002662 & 1.155478 & 1.023546 & 0.921304 & 0.823793 & 0.748430 \\
\hline
\end{tabular}


The $\varphi_{\mathrm{v}}$ versus $(\sqrt{ } \mathrm{m})$ data in the low concentration region is shown (Table 1) sudden change in $\varphi_{v}$ value at particular molality (Fig. 3-4). In premicellar region, the molecules exist as monomers and the monomer interaction may account for $\varphi_{\mathrm{v}}$ with concentration having the free space between the molecules. The limiting apparent molar volume $\left(\varphi_{\mathrm{v}}^{0}\right)$ and $\mathrm{S}_{\mathrm{v}}$ values

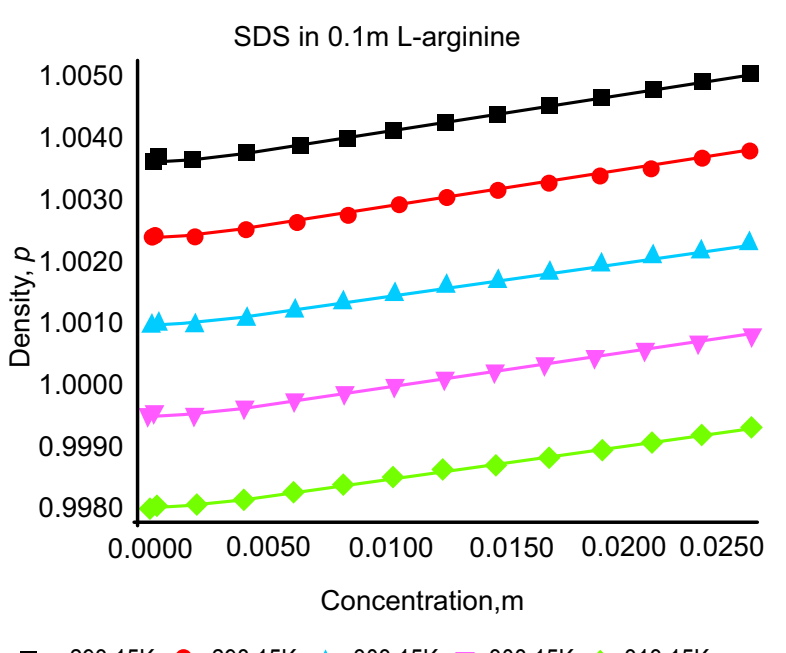

$293.15 K-298.15 K-303.15 K-308.15 K-313.15 K$

Fig. 1. Plots of density versus concentration for $\mathrm{SDS}$ in $0.1 \mathrm{~m}$ L-arginine solution at different temperatures.

SDS in $0.1 \mathrm{~m}$ 2-amino butyric acid

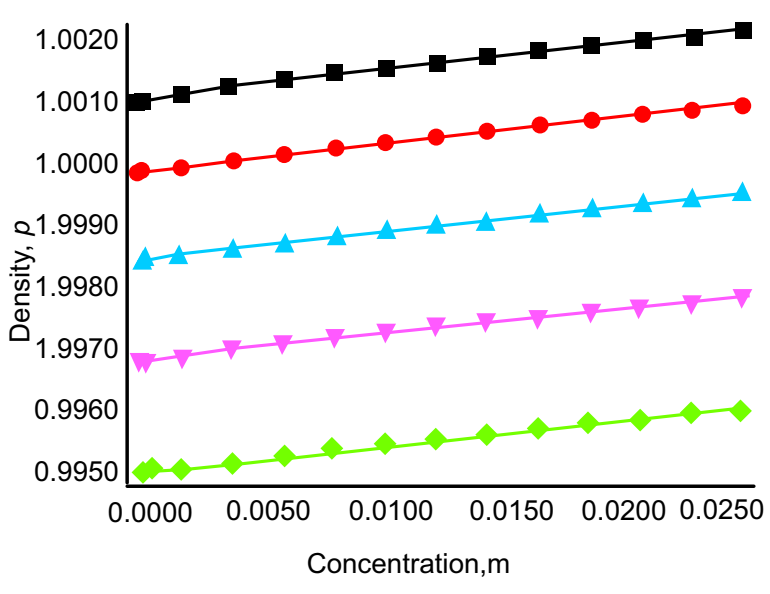

$293.15 \mathrm{~K}-298.15 \mathrm{~K}-303.15 \mathrm{~K}-308.15 \mathrm{~K} \rightleftharpoons 313.15 \mathrm{~K}$

Fig. 2. Plots of density versus concentration for SDS in $0.1 \mathrm{~m} 2$-amino butyric acid solution at different temperatures. along with the standard error, are given in Table 2 . For SDS in aqueous L-arginine and 2-amino butyric acid solution, the values of $S_{\mathrm{v}}$ are positive which indicates the very strong ion-ion interactions in this region. In both cases, the ion-ion interaction is greater of the premicellar region than the post micellar region.

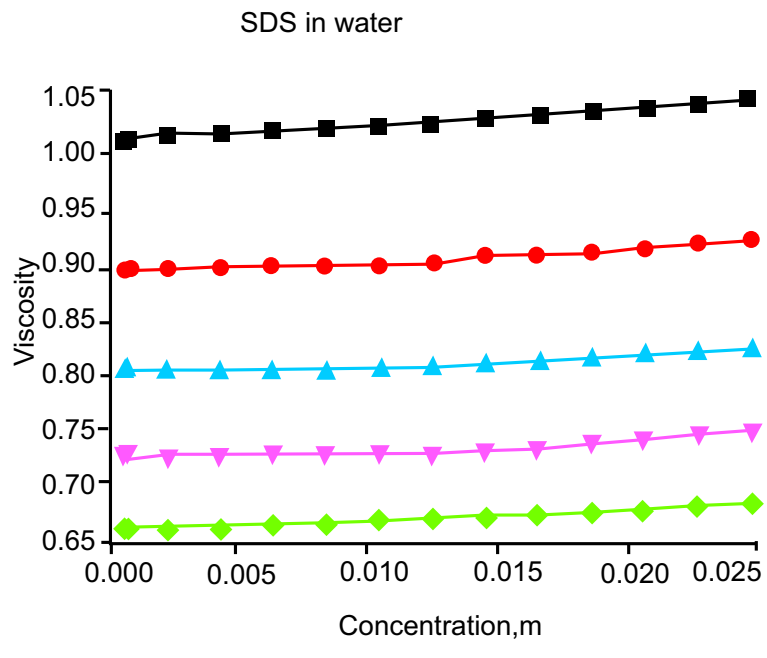

$293.15 \mathrm{~K}-298.15 \mathrm{~K} \leftarrow 303.15 \mathrm{~K}-308.15 \mathrm{~K} \leadsto-313.15 \mathrm{~K}$

Fig. S1. Plots of viscosity versus concentration for SDS in water at different temperatures.

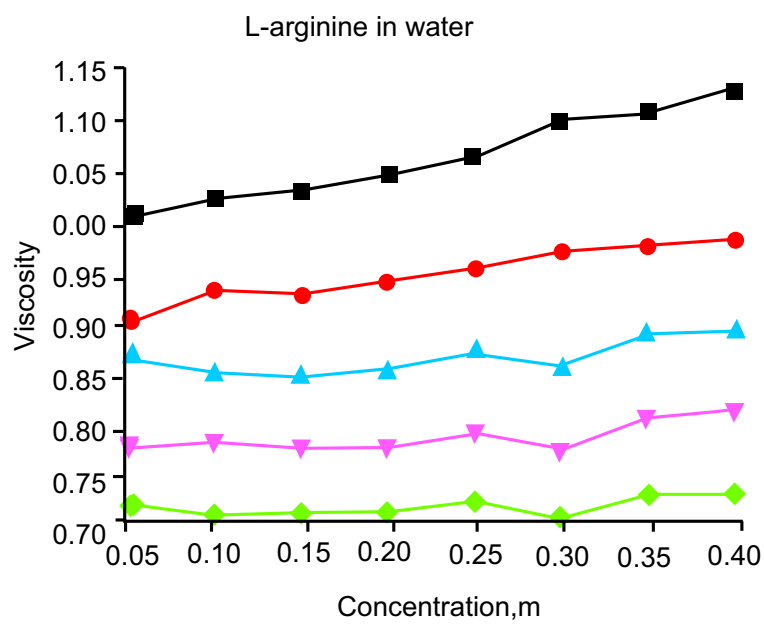

$293.15 \mathrm{~K}-298.15 \mathrm{~K}-303.15 \mathrm{~K}-308.15 \mathrm{~K} \leadsto 313.15 \mathrm{~K}$

Fig. S2. Plots of viscosity versus concentration for L-arginine in water at different temperatures. 
Table 2. Limiting apparent molar volumes, $\varphi_{\mathrm{v}}^{0}$ and experimental slopes, $\mathrm{S}_{\mathrm{v}}$ for binary and ternary solutions at different temperatures

\begin{tabular}{|c|c|c|c|c|}
\hline Compound & $\mathrm{T} / \mathrm{K}$ & $\varphi_{\mathrm{v}}^{0} / \mathrm{m}^{3} / \mathrm{mol} / 10^{6}$ & $\mathrm{~S}_{\mathrm{V}} / \mathrm{m}^{3} / \mathrm{mol} / 10^{-6}$ & $\left(\partial^{2} \varphi^{0}{ }_{\mathrm{v}} / \partial \mathrm{T}^{2}\right)_{\mathrm{r}}$ \\
\hline & & & r region & \\
\hline & 293.15 & $220.05( \pm 0.5238)$ & $67.84( \pm 7.9035)$ & 0.0446 \\
\hline & 298.15 & $220.66( \pm 0.5768)$ & $111.07( \pm 8.7033)$ & \\
\hline & 303.15 & $227.76( \pm 0.4716)$ & $75.63( \pm 7.1164)$ & \\
\hline & 308.15 & $229.79( \pm 0.4348)$ & $109.06( \pm 6.5611)$ & \\
\hline & 313.15 & $240.75( \pm 0.2537)$ & $59.77( \pm 3.8282)$ & \\
\hline \multirow[t]{6}{*}{ SDS + Water } & \multicolumn{4}{|c|}{ Postmicellar region } \\
\hline & 293.15 & $217.30( \pm 1.1761)$ & $110.79( \pm 8.7662)$ & 0.0575 \\
\hline & 298.15 & $218.46( \pm 1.9780)$ & $128.01( \pm 14.743)$ & \\
\hline & 303.15 & $220.15( \pm 2.1387)$ & $145.48( \pm 15.941)$ & \\
\hline & 308.15 & $222.56( \pm 1.5538)$ & $176.68( \pm 11.581)$ & \\
\hline & 313.15 & $233.43( \pm 0.6396)$ & $133.12( \pm 4.7671)$ & \\
\hline \multirow[t]{5}{*}{ L-arginine + Water } & 293.15 & $132.04( \pm 0.0337)$ & $1.32( \pm 0.0711)$ & -0.0009 \\
\hline & 298.15 & $132.35( \pm 0.0328)$ & $1.18( \pm 0.0692)$ & \\
\hline & 303.15 & $132.60( \pm 0.0351)$ & $1.32( \pm 0.0739)$ & \\
\hline & 308.15 & $132.87( \pm 0.0267)$ & $1.21( \pm 0.0562)$ & \\
\hline & 313.15 & $133.00( \pm 0.0306)$ & $1.23( \pm 0.0646)$ & \\
\hline \multirow[t]{11}{*}{ 2-Amino butyric acid + Water } & 293.15 & $75.00( \pm 0.0177)$ & $0.63( \pm 0.0373)$ & 0.0001 \\
\hline & 298.15 & $75.22( \pm 0.0186)$ & $0.81( \pm 0.0394)$ & \\
\hline & 303.15 & $75.49( \pm 0.0253)$ & $0.86( \pm 0.0535)$ & \\
\hline & 308.15 & $75.73( \pm 0.0243)$ & $0.90( \pm 0.0513)$ & \\
\hline & 313.15 & $75.98( \pm 0.0233)$ & $0.86( \pm 0.0492)$ & \\
\hline & \multicolumn{4}{|c|}{ Premicellar region } \\
\hline & 293.15 & $221.74( \pm 0.4523)$ & $24.43( \pm 6.8246)$ & -0.0028 \\
\hline & 298.15 & $221.80( \pm 0.4956)$ & $35.68( \pm 7.4783)$ & \\
\hline & 303.15 & $222.07( \pm 0.5797)$ & $38.48( \pm 8.7474)$ & \\
\hline & 308.15 & $222.14( \pm 0.7381)$ & $55.84( \pm 11.137)$ & \\
\hline & 313.15 & $221.82( \pm 0.6106)$ & $74.57( \pm 9.2139)$ & \\
\hline \multirow{12}{*}{ SDS $+0.10 \mathrm{~m}$ L-arginine + Water } & \multicolumn{4}{|c|}{ Postmicellar region } \\
\hline & 293.15 & $222.48( \pm 0.2755)$ & $15.13( \pm 2.0540)$ & 0.0183 \\
\hline & 298.15 & $222.75( \pm 0.1988)$ & $22.54( \pm 1.4822)$ & \\
\hline & 303.15 & $220.43( \pm 0.7155)$ & $49.83( \pm 5.3347)$ & \\
\hline & 308.15 & $220.85( \pm 0.9753)$ & $58.06( \pm 7.2713)$ & \\
\hline & 313.15 & $222.95( \pm 0.3937)$ & $55.70( \pm 2.9349)$ & \\
\hline & \multicolumn{4}{|c|}{ Premicellar region } \\
\hline & 293.15 & $227.52( \pm 3.5840)$ & $49.54( \pm 54.078)$ & 0.0144 \\
\hline & 298.15 & $223.71( \pm 3.6108)$ & $116.20( \pm 54.4825)$ & \\
\hline & 303.15 & $220.17( \pm 3.4390)$ & $181.83( \pm 51.890)$ & \\
\hline & 308.15 & $227.21( \pm 3.5179)$ & $117.68( \pm 53.081)$ & \\
\hline & 313.15 & $220.63( \pm 4.1196)$ & $219.68( \pm 62.1597)$ & \\
\hline \multirow{6}{*}{$\begin{array}{l}\text { SDS }+0.10 \mathrm{~m} \text { 2-Amino } \\
\text { butyric acid }+ \text { Water }\end{array}$} & \multicolumn{4}{|c|}{$\begin{array}{l}\text { Postmicellar region } \\
\end{array}$} \\
\hline & 293.15 & $224.67( \pm 0.5991)$ & $96.06( \pm 4.4667)$ & 0.0027 \\
\hline & 298.15 & $226.60( \pm 0.6169)$ & $93.36( \pm 4.5990)$ & \\
\hline & 303.15 & $228.76( \pm 0.6510)$ & $89.34( \pm 4.8536)$ & \\
\hline & 308.15 & $229.11( \pm 0.7813)$ & $98.87( \pm 5.8248)$ & \\
\hline & 313.15 & $232.42( \pm 0.6532)$ & $84.09( \pm 4.8700)$ & \\
\hline
\end{tabular}


Table S2. Concentration dependence of viscosity for different ternary solutions at $293.15 \mathrm{~K}, 298.15 \mathrm{~K}, 303.15$ $\mathrm{K}, 308.15 \mathrm{~K}$, and $313.15 \mathrm{~K}$

\begin{tabular}{|c|c|c|c|c|c|c|c|c|c|c|}
\hline \multirow{2}{*}{$\begin{array}{l}\text { Conc./m/ } \\
\mathrm{mol} / \mathrm{kg}\end{array}$} & \multicolumn{10}{|c|}{ Viscosity, $\eta / \mathrm{kg} / \mathrm{m} / \mathrm{s} / 10^{2}$} \\
\hline & $293.15 \mathrm{~K}$ & $298.15 \mathrm{~K}$ & $303.15 \mathrm{~K}$ & $308.15 \mathrm{~K}$ & $313.15 \mathrm{~K}$ & $293.15 \mathrm{~K}$ & $298.15 \mathrm{~K}$ & $303.15 \mathrm{~K}$ & $308.15 \mathrm{~K}$ & $313.15 \mathrm{~K}$ \\
\hline \multicolumn{6}{|c|}{ SDS in $0.1 \mathrm{~m}$ aqueous L-arginine solution } & \multicolumn{5}{|c|}{ SDS in $0.1 \mathrm{~m}$ aqueous 2 -amino butyric acid solution } \\
\hline 0.0003 & 1.005766 & 0.926794 & 0.836097 & 0.760695 & 0.683254 & 1.005056 & 0.894653 & 0.828575 & 0.723083 & 0.684365 \\
\hline 0.0005 & 1.005562 & 0.927794 & 0.839057 & 0.761171 & 0.683763 & 1.005085 & 0.894776 & 0.823797 & 0.723835 & 0.678512 \\
\hline 0.0020 & 1.006412 & 0.928261 & 0.841114 & 0.761322 & 0.685855 & 1.005206 & 0.895308 & 0.809071 & 0.723641 & 0.664141 \\
\hline 0.0040 & 1.008132 & 0.929944 & 0.842451 & 0.762489 & 0.687359 & 1.005350 & 0.896375 & 0.806566 & 0.724578 & 0.661281 \\
\hline 0.0060 & 1.007273 & 0.932388 & 0.844677 & 0.763843 & 0.688760 & 1.005459 & 0.897265 & 0.805294 & 0.724721 & 0.660462 \\
\hline 0.0080 & 1.011818 & 0.934520 & 0.845889 & 0.768876 & 0.690148 & 1.005651 & 0.897719 & 0.805850 & 0.724608 & 0.660438 \\
\hline 0.0100 & 1.008638 & 0.937186 & 0.848522 & 0.769693 & 0.690577 & 1.005713 & 0.899603 & 0.805604 & 0.727675 & 0.661517 \\
\hline 0.0120 & 1.014986 & 0.940476 & 0.851224 & 0.770383 & 0.691054 & 1.006123 & 0.903565 & 0.809245 & 0.732172 & 0.665179 \\
\hline 0.0140 & 1.016533 & 0.943789 & 0.855782 & 0.771853 & 0.695315 & 1.006685 & 0.907504 & 0.814156 & 0.737671 & 0.669622 \\
\hline 0.0160 & 1.019022 & 0.947180 & 0.858583 & 0.773283 & 0.695727 & 1.007206 & 0.911099 & 0.819397 & 0.743582 & 0.673641 \\
\hline 0.0180 & 1.008286 & 0.949096 & 0.860820 & 0.775120 & 0.697103 & 1.007921 & 0.915365 & 0.826518 & 0.750632 & 0.680325 \\
\hline 0.0200 & 1.017327 & 0.950937 & 0.862739 & 0.780617 & 0.698751 & 1.008780 & 0.919444 & 0.834076 & 0.756953 & 0.687196 \\
\hline 0.0220 & 1.018100 & 0.953410 & 0.864178 & 0.782258 & 0.699664 & 1.009767 & 0.924209 & 0.843394 & 0.765377 & 0.696557 \\
\hline 0.0240 & 1.023993 & 0.956160 & 0.866490 & 0.784292 & 0.700959 & 1.010863 & 0.929703 & 0.853413 & 0.774554 & 0.705047 \\
\hline
\end{tabular}

The measured viscosities of binary and ternary solutions are listed in Tables S1 and Table S2 respectively as functions of concentration and temperature. The viscosities have been found to increase with the increase in the molal concentration (Fig. S1-S3). The viscometer was immersed in a transparent thermostat bath with the precision of the set temperature better than $0.01 \mathrm{~K}$. Each measurement was automatically repeated at least five times and yielded a reproducibility of the flow time

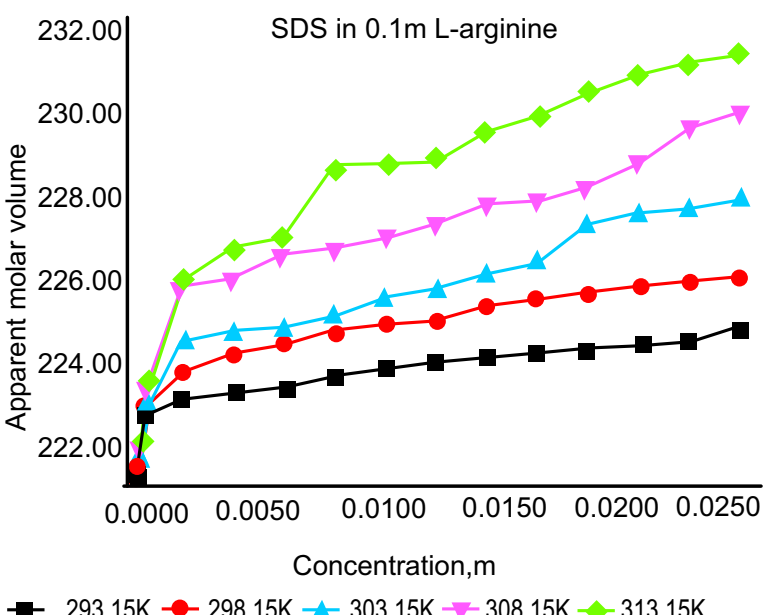

Fig. 3. Plots of apparent molar volume versus concentration for SDS in $0.1 \mathrm{~m} \mathrm{~L}$-arginine solution at different temperatures. of better than $0.02 \%$ (Renato et al., 2015). The kinematic viscosity of solutions, $v\left(\mathrm{~m}^{2} \mathrm{~s}^{-1}\right)$, was calculated from the Poiseuille equation:

$$
\eta=\mathrm{A} \rho \mathrm{t}
$$

where:

$\mathrm{A}=\pi \mathrm{r}^{4} \mathrm{hg} / 8 \mathrm{lv}$ is the viscometer constant at a particular temperature determined by measuring the flow time of

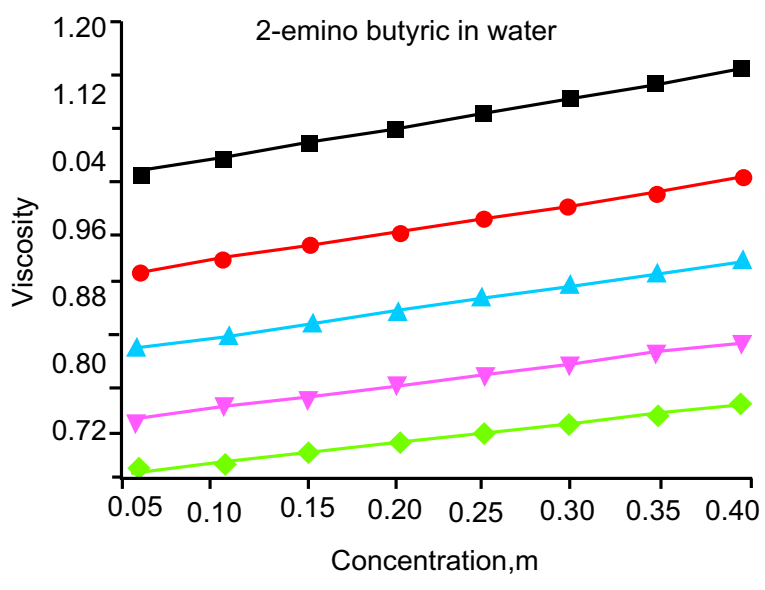

$293.15 \mathrm{~K}-298.15 \mathrm{~K}-303.15 \mathrm{~K}-308.15 \mathrm{~K} \neg-313.15 \mathrm{~K}$

Fig. S3. Plots of viscosity versus concentration for 2-amino butyric acid in water at different temperatures. 
Table 3. The viscosity coefficient values A, B and dB/dT for binary and ternary solutions as a function of temperature

\begin{tabular}{|c|c|c|c|c|}
\hline Compound & $\mathrm{T} / \mathrm{K}$ & A-Coefficient & B-Coefficient & $(\mathrm{dB} / \mathrm{dT})$ \\
\hline \multirow[t]{12}{*}{ SDS + Water } & \multicolumn{4}{|l|}{ Premicellar region } \\
\hline & 293.15 & $-17.0183( \pm 12.344)$ & $1.3916( \pm 0.8181)$ & -0.03579 \\
\hline & 298.15 & $6.8905( \pm 3.5124)$ & $-0.5460( \pm 0.2328)$ & \\
\hline & 303.15 & $4.4649( \pm 7.6918)$ & $0.0411( \pm 0.5098)$ & \\
\hline & 308.15 & $7.5908( \pm 7.5654)$ & $-0.1941( \pm 0.5014)$ & \\
\hline & 313.15 & $1.6932( \pm 8.2923)$ & $0.3210( \pm 0.5496)$ & \\
\hline & \multicolumn{4}{|l|}{ Postmicellar region } \\
\hline & 293.15 & $4.6520( \pm 1.4057)$ & $-0.5841( \pm 0.1886)$ & 0.09630 \\
\hline & 298.15 & $6.1267( \pm 3.5265)$ & $-0.6375( \pm 0.4731)$ & \\
\hline & 303.15 & $-0.7018( \pm 2.5951)$ & $0.4973( \pm 0.3482)$ & \\
\hline & 308.15 & $-3.3606( \pm 3.3720)$ & $0.8967( \pm 0.4390)$ & \\
\hline & 313.15 & $-4.1326( \pm 3.6037)$ & $1.0564( \pm 0.4835)$ & \\
\hline \multirow[t]{5}{*}{ L-arginine + Water } & 293.15 & $0.6913( \pm 0.1398)$ & $0.0697( \pm 0.0339)$ & 0.01150 \\
\hline & 298.15 & $0.2261( \pm 0.0864)$ & $0.2271( \pm 0.0217)$ & \\
\hline & 303.15 & $0.2023( \pm 0.0953)$ & $0.2596( \pm 0.0240)$ & \\
\hline & 308.15 & $0.1769( \pm 0.0606)$ & $0.2714( \pm 0.0153)$ & \\
\hline & 313.15 & $-0.0135( \pm 0.0949)$ & $0.3349( \pm 0.0239)$ & \\
\hline \multirow[t]{5}{*}{ 2-Amino butyric acid + Water } & 293.15 & $-0.2037( \pm 0.0547)$ & $0.4405( \pm 0.0138)$ & -0.00572 \\
\hline & 298.15 & $0.0788( \pm 0.0267)$ & $0.3363( \pm 0.0067)$ & \\
\hline & 303.15 & $-0.0560( \pm 0.0198)$ & $0.3969( \pm 0.0050)$ & \\
\hline & 308.15 & $0.3226( \pm 0.0887)$ & $0.2451( \pm 0.0223)$ & \\
\hline & 313.15 & $0.0268( \pm 0.0084)$ & $0.3431( \pm 0.0021)$ & \\
\hline \multirow[t]{12}{*}{$\mathrm{SDS}+0.10 \mathrm{~m}$ L-arginine + Water } & \multicolumn{4}{|l|}{ Premicellar region } \\
\hline & 293.15 & $-17.4747( \pm 7.0642)$ & $2.0007( \pm 0.4682)$ & 0.05810 \\
\hline & 298.15 & $-9.6749( \pm 5.2065)$ & $1.5815( \pm 0.3451)$ & \\
\hline & 303.15 & $-12.754( \pm 7.4737)$ & $1.8068( \pm 0.4953)$ & \\
\hline & 308.15 & $-11.993( \pm 2.0755)$ & $2.4283( \pm 0.1376)$ & \\
\hline & 313.15 & $-19.980( \pm 5.2205)$ & $3.0300( \pm 0.3460)$ & \\
\hline & \multicolumn{4}{|l|}{ Postmicellar region } \\
\hline & 293.15 & $-4.3341( \pm 6.3374)$ & $1.2485( \pm 0.8502)$ & 0.03443 \\
\hline & 298.15 & $-1.7896( \pm 5.8111)$ & $0.9193( \pm 0.7796)$ & \\
\hline & 303.15 & $0.5501( \pm 3.4739)$ & $0.5871( \pm 0.4661)$ & \\
\hline & 308.15 & $-10.865( \pm 3.4491)$ & $2.2988( \pm 0.4627)$ & \\
\hline & 313.15 & $-4.4896( \pm)$ & $1.4194( \pm 0.8383)$ & \\
\hline $\mathrm{SDS}+0.10 \mathrm{~m} 2$-Amino & Premicellar region & & & \\
\hline \multirow[t]{11}{*}{ butyric acid + Water } & 293.15 & $-1.3658( \pm 0.2589)$ & $0.1916( \pm 0.0172)$ & 0.00064 \\
\hline & 298.15 & $0.0289( \pm 0.1208)$ & $0.0513( \pm 0.0080)$ & \\
\hline & 303.15 & $-1.2646( \pm 0.2942)$ & $0.1866( \pm 0.0195)$ & \\
\hline & 308.15 & $0.8191( \pm 0.1900)$ & $0.0049( \pm 0.0126)$ & \\
\hline & 313.15 & $-1.6660( \pm 0.3782)$ & $0.2308( \pm 0.0251)$ & \\
\hline & \multicolumn{4}{|l|}{ Postmicellar region } \\
\hline & 293.15 & $3.2760( \pm 0.1612)$ & $-0.2711( \pm 0.0216)$ & 0.000769 \\
\hline & 298.15 & $3.4458( \pm 0.1048)$ & $-0.2793( \pm 0.0141)$ & \\
\hline & 303.15 & $3.4848( \pm 0.0507)$ & $-0.2695( \pm 0.0068)$ & \\
\hline & 308.15 & $3.3555( \pm 0.0600)$ & $-0.2617( \pm 0.0080)$ & \\
\hline & 313.15 & $3.4081( \pm 0.2682)$ & $-0.2607( \pm 0.0360)$ & \\
\hline
\end{tabular}




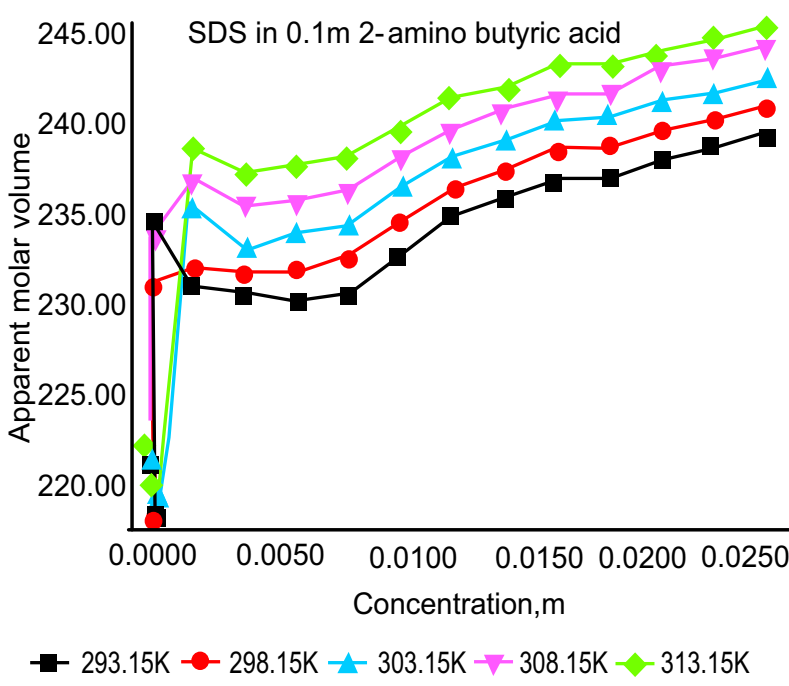

Fig. 4. Plots of apparent molar volume versus concentration for SDS in $0.1 \mathrm{~m} 2$-amino butyric acid solution at different temperatures.

water. $\rho$ is the density of investigating solution, which was determined at each temperature $\pm 0.005 \mathrm{~K}$ uses Anton Paar density meter DMA 5000 (Anton Paar, Graz, Austria) with a declared reproducibility $\sim 5 \times 10^{-3}$ $\mathrm{kg} / \mathrm{m}^{3}$. The calculated viscosity for water $\left(\eta_{\mathrm{o}}\right)$ are $1.0050,0.8937,0.8007,0.7225$ and 0.6560 at 293.15 $\mathrm{K}, 298.15 \mathrm{~K}, 303.15 \mathrm{~K}, 308.15 \mathrm{~K}$ and $313.15 \mathrm{~K}$, respectively.

To calculate the B-coefficient values, the viscosity data have been analyzed in terms of the semi-empirical Jones-Dole equation arranging into the straight line from given below (Jones and Dole, 1929):

$$
\text { For electrolyte } \frac{\eta}{\eta_{\circ}}=1+A \sqrt{c}+B C
$$

or

$$
\frac{\eta}{\sqrt{c}}=1+\mathrm{A}+\mathrm{B} \sqrt{ } \mathrm{C}
$$

where:

$\eta$ and $\eta_{\circ}$ are the viscosities of the solution and solvent, respectively.

At higher concentration ( $c>0.5 \mathrm{~mol} \mathrm{dm}^{-3}$ ) a quadratic term $\mathrm{Dc}^{2}$ is added in eq. (4) and that expression is known as Kaminsky relation. The viscosity A-coefficient is a measure of long-range ion-ion interactions, and the viscosity B-coefficient depends on the ion-solvent interactions (Marcus, 2010). The A and B coefficients can be obtained from the intercept and the slope of the straight line using equation (4) in the next form.
The B-coefficient represents information regarding solute-solvent interaction and shape and size effect on the solvent structure (Feakins et al., 1974). The calculated values of the B-coefficient are represented in Table 3. The sign of $\frac{d B}{d T}$ i.e. first derivative of the viscosity coefficient of $\mathrm{B}$ with respect to temperature, which corresponds to structure making, or breaking properties of solutes was determined (Zhenning et al., 2004).

For aqueous L-Arginine and 2-amino butyric acid solutions, the Table 3 report that the values of $\frac{\mathrm{dB}}{\mathrm{dT}}$ have positive \& negative which corresponds to structure breaking \& making behaviour, respectively. For SDS in aqueous solution, $\frac{\mathrm{dB}}{\mathrm{dT}}$ has been found to be negative in premicellar and positive in postmicellar region suggesting structure making \& breaking properties, respectively. It is seen that $\frac{\mathrm{dB}}{\mathrm{dT}}$ is positive for SDS in both aqueous amino acid solutions in the premicellar and postmicellar region. This indicates that SDS acts as structure breaker for both regions in aqueous L-arginine and 2-amino butyric acid solvent system.

\section{Conclusion}

The studied on the solution properties of SDS, LArginine in aqueous solution and SDS in mixed solutions reveal the following: The behaviour of SDS in aqueous solution is a temperature depends properly. In premicellar and postmicellar region, it appears to be structure making and breaking properties respectively at the studied temperatures. Aqueous L-arginine and 2-amino butyric acid solutions appear to structure breaking \& making behavior respectively. It is seen that SDS acts as structure breaker for both regions in aqueous L-arginine and 2amino butyric acid solvent system.

\section{Acknowledgement}

The authors are grateful to the Chemistry Discipline, Khulna University, Bangladesh, for providing financial support and laboratory facilities to carry out this research work.

Conflict of Interest. The authors declare that there is no conflict of interest.

\section{References}

Debye, P., Anacker, E.W. 1951. Micelle shape from dissymmetry measurements. Journal of Physical Chemistry, 55: 644-655.

Desnoyers, J.E. 2009. Structural effects in aqueous 
solutions: a thermodynamic approach. Pure Applied Chemistry, 54: 1469-1478.

Feakins, D., Freemantle, D.J., Lawrence, K.G. 1974. Transition state treatment of the relative viscosity of electrolytic solutions. Applications to aqueous, non-aqueous and methanol + water systems. Journal of Chemical Society Faraday Translation 1, 70: 795-806.

George, N., Harold, A.S. 1962. Structure of water and hydrophobic bonding in proteins. II model for the thermodynamic properties of aqueous solutions of hydrocarbons. The Journal of Chemical Physics, 36: 3382-3401.

Grinnell Jones, Malcolm Dole. 1929. The viscosity of aqueous solutions of strong electrolytes with special reference to barium chloride. Journal of American Chemical Society, 51: 2950-2954.

Hedwig, G.R., Reading, J.F., Lilley, T.H. 1991. Aqueous solutions containing amino acids and peptides. Part 27. Partial molar heat capacities and partial molar volumes of some $\mathrm{N}$-acetyl amino acid amides, some $N$-acetyl peptide amides and two peptides at $25^{\circ} \mathrm{C}$. Journal of Chemical Society Faraday Translation, 87: 1751-1758.

IUPAC-IUBMB Joint Commission on Biochemical Nomenclature, 2007. "Nomenclature and Symbolism for Amino acids and Peptides". Recommendations on Organic \& Biochemical Nomenclature, Symbols \& Terminology etc.

John, L., Speier. 1952. The preparation and properties of (hydroxyorgano)-silanes and related compounds. Journal of American Chemical Society, 74: 10031010.

Jones, G., Dole, M. 1929. The viscosity of aqueous solutions of strong electrolytes with special reference to barium chloride. Journal of Chemical Society, 51: 2950-2964.

Joshua, A., Blake, L., Rankin, M., Ben-Amotz, D. 2015. Micelle structure and hydrophobic hydration, Journal of American Chemical Society, 137: 10809 10815.

Kabiraz, D.C., Biswas, T.K., Islam, M.N., Huque, M.E. 2011. Studies on molecular interactions of some electrolytes in water by volumetric and viscometric measurements at $\mathrm{T}=(303.15$ to $323.15 \mathrm{~K})$. Journal of Scientific Research, 3: 437-444.

Mahendra, N.R., Rajani, D., Pran, K.R., Debabrata, B. 2010. Apparent molar volumes and viscosity $B$ - coefficients of carbohydrates in aqueous cetrimonium bromide solutions at $(298.15,308.15$ and $318.15 \mathrm{~K})$. Journal of Chemical and Engineering Data, 55: 3617-3624.

Marcus, Y. 2010. Effect of ions on the structure of water. Pure Applied Chemistry, 82: 1889-1899.

Masanori Inui, Shinichi Takeda, Tetsuo Uechi. 1993. Ultrasonic velocity and density measurements of molten $\mathrm{CuCl}-\mathrm{CuBr}$ mixtures. Journal of Physical Society Japan, 62: 3142-3149.

Masson, D.O. 1929. Solute molecular volumes in relation to solvation and ionization. Philosphical Magazine, 8: $218-235$.

Niki, S., Jha Nand, K. 2011. Thermodynamic studies on the interaction of folic acid with bovine serum albumin. The Journal of Chemical Thermodynamics, 43: 814-821.

Qiu, X., Lei, Q., Fang, W., Lin, R. 2008. A calorimetric study on interactions of amino acids with sodium dodecylsulfate and dodecyl tri-methylammonium bromide in aqueous solutions at $298.15 \mathrm{~K}$. Thermochimica Acta, 478: 54-56.

Renato, T., Toni, J., Marija Bester-Rogak. 2015. Viscosity $B$-coefficient for sodium chloride in aqueous mixtures of 1,4-dioxane at different temperatures. Acta Chimica Slovenica, 62: 531-537.

Shashank, D., Jagdish, C.A. 2001. Interaction of bovine serum albumin with anionic surfactants. Physical Chemistry Chemical Physics, 3: 4583-4591.

Singh, S.K., Kundu, A., Kishore, N. 2004. Interactions of some amino acids and glycine peptides with aqueous sodium dodecyl sulfate and cetyl trimethylammonium bromide at $\mathrm{T}=298.15 \mathrm{~K}$ : a volumetric approach. Journal of Chemical Thermodynamics, 36: 7-16.

Tapiero, H., Mathé, G., Couvreur, P., Tew, K.D. 2002. "L-Arginine". (review). Biomedicine and Pharmacotherapy, 56: 439-445.

Wu, G., Jaeger, I.A., Bazer, F.W., Rhoads, J.M. 2004. "Arginine deficiency in preterm infants: biochemical mechanisms and nutritional implications". (review). The Journal of Nutritional Biochemistry, 15: 442451.

Yan, Z., Wang, J., Kong, W., Lu, J. 2004. Effect of temperature on volumetric and viscosity properties of some amino acids in aqueous calcium chloride solution. Fluid Phase Equilibria, 215: 143-150. 